Pathologe 2010 · [Suppl 2] 31:211-214

DOI 10.1007/s00292-010-1340-z

Online publiziert: 15. August 2010

(c) Springer-Verlag 2010

\author{
A.V. Rüsseler ${ }^{1}$ B. Brors ${ }^{2}$. T. Fischer ${ }^{3}$. J.T. Hartmann ${ }^{4}$. W. Hartmann ${ }^{1}$.

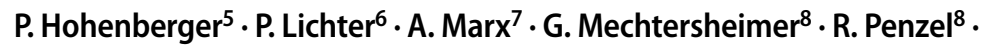 \\ M. Renner ${ }^{8} \cdot$ H. - U. Schildhaus ${ }^{1} \cdot$ P. Schirmacher ${ }^{8} \cdot$ E. Sievers ${ }^{1} \cdot$ P. Ströbel ${ }^{7}$. \\ E. Wardelmann ${ }^{1} \cdot$ E. Ziesché $^{9} \cdot$ R. Büttner ${ }^{1}$ \\ ${ }^{1}$ Institut für Pathologie, Universitätsklinikum Bonn \\ ${ }^{2}$ Abteilung für Theoretische Bioinformatik, \\ Deutsches Krebsforschungszentrum Heidelberg \\ ${ }^{3}$ Universitätsklinik für Hämatologie und Onkologie \\ der Otto-von-Guericke-Universität Magdeburg \\ ${ }^{4}$ Medizinische Klinik und Poliklinik, UKT-Universitätsklinikum Tübingen \\ der Eberhard-Karls-Universität, Tübingen \\ ${ }^{5}$ Chirurgische Klinik, Universitätsmedizin Mannheim \\ der Universität Heidelberg, Mannheim \\ ${ }^{6}$ Abteilung Molekulargenetik, Deutsches Krebsforschungszentrum Heidelberg \\ ${ }^{7}$ Pathologisches Institut, Universitätsmedizin Mannheim \\ der Universität Heidelberg, Mannheim \\ ${ }^{8}$ Institut für Pathologie, Universität Heidelberg \\ ${ }^{9}$ III. Medizinische Klinik und Poliklinik der Universitätsmedizin Mainz
}

\title{
Molekularpathologie von Sarkomen
}

\section{Erste Ergebnisse des Sarkomforschungs- verbundes KoSar}

Sowohl die Diagnostik als auch die Behandlung von Weichteilsarkomen sind nach wie vor eine große Herausforderung in der klinischen Praxis. Sarkome sind seltene Tumoren, die eine heterogene Gruppe verschiedener Entitäten mit sehr unterschiedlichem klinischem Verhalten, von lokal aggressiv bis hochmaligne mit schneller systemischer Streuung, darstellen. Die konventionelle histopathologische Diagnostik beruht auf dem Konzept der Histogenese, in dem von der Differenzierung der Tumorzellen Rückschlüsse auf die mutmaßliche Ursprungszelle gezogen werden. Allerdings kann in vielen Fällen die Definition der Differenzierung allein durch die Mittel der (Immun-)Histologie fehlleitend sein. So wird eine Expression von Desmin auch in vielen Sarkomen ohne myogene Differenzierung beobachtet. Hinzu kommt, dass bei manchen Entitäten die Ursprungszelle noch unbekannt ist. Man weiß z. B. bei malignen fibrösen Histiozytomen (MFH) bezüglich ihrer Herkunft nur, dass sie sicherlich nicht histiozytären Ursprungs sind und diese Tumorgruppe wahrscheinlich verschiedene Entitäten umfasst.

Zudem befunden viele pathologische Institute insgesamt nur wenige Sarkome, und daher bleibt in der klinischen Praxis die präzise Diagnose und die richtige Behandlung bei Erstmanifestation ein bedeutendes Problem. Basierend auf den Daten unseres Referenzzentrums schätzen wir, dass mehr als 30\% der Sarkome reklassifiziert werden müssten.

Sarkome tragen in vielen Fällen spezifische molekulare Veränderungen, und eine genaue Klassifikation beruht daher auf der Ermittlung der zugehörigen molekularen Aberrationen. Zum Beispiel ist für dedifferenzierte Liposarkome eine hochgradige $M D M 2$-Cluster-Amplifikation charakteristisch, die auch immunhistochemisch darstellbar ist (• Abb. 1). Ag- gressive Fibromatosen (Desmoidtumoren) sind durch ihre aktivierenden $\mathrm{Mu}$ tationen im WNT-Signal-Transduktionsweg gut definiert. Ein wichtiger Beitrag zur Sarkomdiagnostik ist daher von umfassenden molekularen Analysen von definierten und gut charakterisierten Sarkomgewebesammlungen zu erwarten.

Zusammengefasst sind Sarkome seltene Tumoren, die eine umfassende Expertise in der Diagnostik und in molekularen Techniken benötigen und daher am besten in spezialisierten Zentren diagnostiziert und behandelt werden. Um diese Situation zu verbessern, hat sich mit Förderung durch die Deutsche Krebshilfe (DKH) der Sarkomforschungsverbund KoSar (Kompetenznetz Sarkome) gebildet.

Die genauen Ziele des KoSar-Verbundes sind:

- Aufbau eines Archivs von präzise diagnostizierten Sarkomen, 


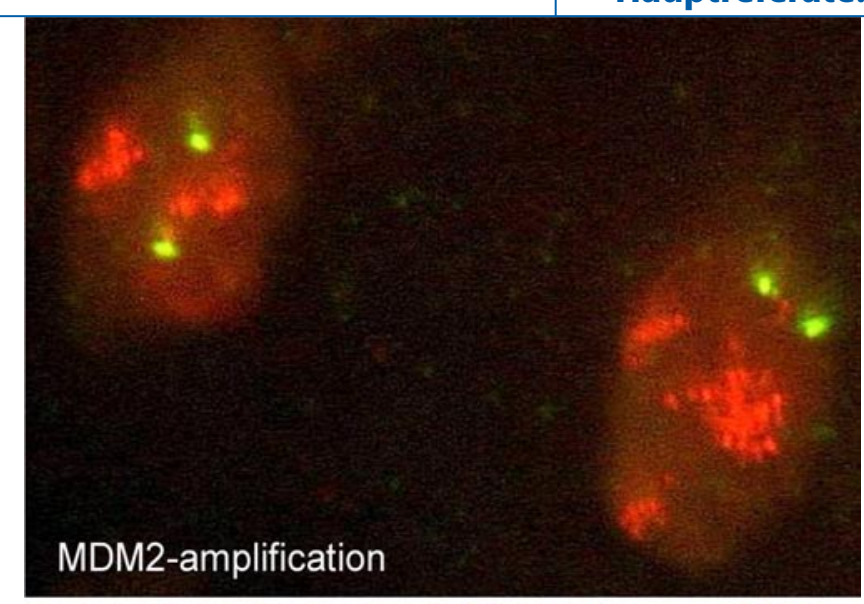

\section{Hauptreferate: Molekulare Tumorpathologie}

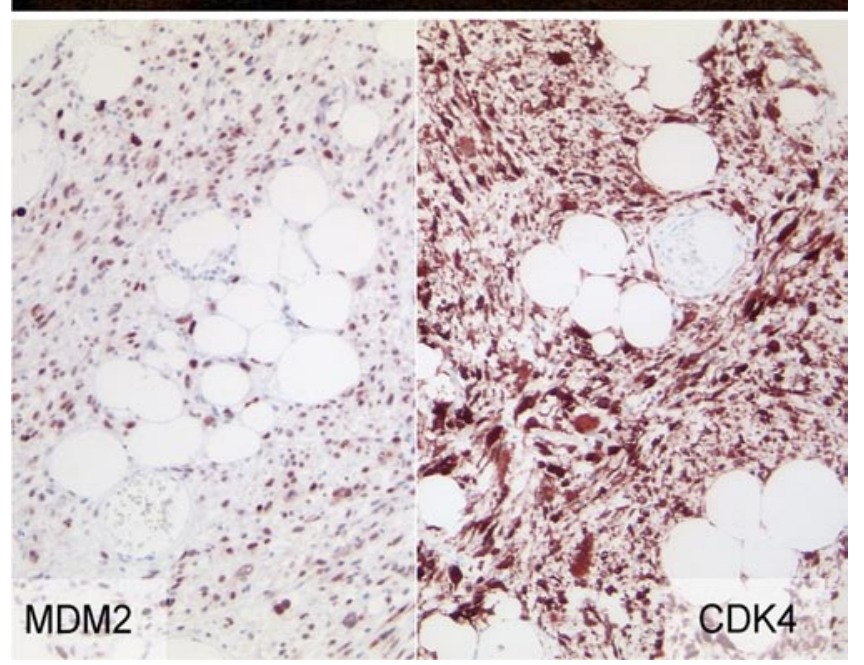

Abb. $1<$ Nachweis einer MDM2-Amplifikation mittels Fluoreszenzin-situ-Hybridisierung (oben) sowie immunhistochemischer Nachweis der nukleären Expression von MDM2 und CDK4 (unten) in Tumorzellen von dedifferenzierten Liposarkomen
- Definition diagnostischer Algorithmen durch genomische Muster von spezifischen molekularen Aberrationen und Genexpressionssignaturen,

- die Suche nach aktivierten Signaltransduktionswegen und molekularen Zielstrukturen/Targets, welche für selektive Therapien verwendet werden könnten.

Es ist schließlich unser Ziel, die Ergebnisse dieser Ansätze in neue klinische Studien $\mathrm{zu}$ transferieren, um zielgerichtete molekulare Therapien zu validieren.

\section{Zusammenfassung erster Ergebnisse des KoSar-Forschungsbundes}

\section{Sarkomarchiv und Referenzzentrum}

Wir haben erfolgreich eine Gewebeanalytik (Plattform 1 - „Standardized tissue analysis and assembly of sarcomas") als standardisiertes Gewebearchiv etabliert und eine Sammlung von gut charakterisierten Sarkomentitäten zusammengestellt.

Innerhalb dieses Gewebearchivs haben wir reichlich formalinfixierte Proben aus allen wichtigen Sarkomentitäten gesammelt und „Tissue-Microarrays“ hergestellt, um diagnostische Algorithmen zu definieren und die immunhistochemischen Profile weiter zu verfeinern, die aus den wissenschaftlichen Teilprojekten und aus den bioinformatischen Analysen aus Plattform 2 („Bioinformatics“) resultieren. Entgegen unseren ursprünglichen Erwartungen haben wir auch genügend frische schockgefrorene Proben erhalten, um alle Screening-Projekte zur Durchführung genomweiter Transkriptome und genetischer Aberrationen zu unterstützen.

Nach Etablierung des Bonn/Heidelberg-Sarkom-Referenzzentrums während der Förderphase haben wir im Jahr 2010 ungefähr 1000 Sarkome zur Referenzdiagnostik einschließlich 580 gastrointestinalen Stromatumoren (GIST) erhalten. Durch die aktuell stark wachsenden Zah- len glauben wir, dass wir 2011 ungefähr $20 \%$ aller klinisch relevanten Sarkome in Deutschland begutachten werden.

Da bis zu 30\% der in peripheren Krankenhäusern gestellten Sarkomdiagnosen eine mehr oder minder umfassende Reklassifizierung brauchen, bedient die KoSar-Referenzpathologie sowohl die wissenschaftlichen Teilprojekte im Rahmen des Forschungskonsortiums als auch aktuelle klinische Bedürfnisse.

\section{Molekulare Definition von Sarkomen, Identifizierung von molekularen Zielstrukturen und vorklinische Validation}

Bisher wurde in Teilprojekt 1 ein genomweites Screening von 80 primären, unbehandelten, „High-grade-Sarkom-Proben“ aus 7 verschiedenen Sarkomentitäten angefertigt. Zurzeit arbeiten wir daran, das Kollektiv mit weiteren Sarkomfällen zu vergrößern. Unter Verwendung des „HumanHT-12 v3 bead array“ (Illumina Inc.) mit genomweiter Transkriptionsabdeckung konnten signifikante Genexpressionssets für synoviale Sarkome, Leiomyosarkome, myxoide Liposarkome und ein kleines Expressionsset für Myxofibrosarkome definiert werden. Eine integrative bioinformatische Auswertung der gesamten Daten wird gerade durchgeführt. Unser Ziel ist es hier, eine diagnostische und/oder prognostische Signatur zu definieren.

Mithilfe des Array-CGH (Agilent 244 K Arrays) wurden an den gleichen Proben wenige, aber dafür spezifische genomische Zugewinne und Verluste in myxoiden Liposarkomen und synovialen Sarkomen identifiziert, die auf interessanteste Regionen mutmaßlicher Tumorsuppressorgene und Onkogene hinweisen.

Im Gegensatz dazu zeigten die malignen fibrösen Histiozytome (MFH) und pleomorphen Liposarkome sehr instabile Genome mit zahlreichen Gengewinnen und -verlusten. Die genomischen Aberrationsmuster wiesen Gemeinsamkeiten, aber auch deutliche Unterschiede auf, was unsere Hypothese unterstützt, dass die MFH keine eigene Entität, sondern eine Art Endpunkt der Dedifferenzierung von verschiedenen Sarkomen mit hoher genetischer Instabilität darstellen. 
Zusammengenommen sind diese Screening-Untersuchungen des KoSarKonsortiums die aktuell größten und systematischsten Analysen, um entitätsspezifische Transkriptome, genomweite $\mathrm{Zu}$ gewinne und Verluste chromosomalen Materials an ein und demselben Kollektiv hochauflösend zu beschreiben. Die Daten bilden die Grundlage für eine große gemeinsame Veröffentlichung aus dem Konsortium und werden viele weitere Studien über ortsspezifische genomische Veränderungen im Zusammenhang mit Veränderungen in der Genexpression anregen, wie z. B. über den PPAR-Signalweg in myxoiden Liposarkomen. Wichtig ist, dass diese Untersuchungen eine weitere Grundlage für die Generierung von Datensätzen bilden, um systematisch nach aktivierten Signalwegen zu suchen und die Genverteilungsmuster mit den Mustern von normalen Zellen, z. B. von mesenchymalen Stammzellen, Fibroblasten und Endothelzellen, sowie der Genverteilung in den Sarkomzelllinien aus Teilprojekt 3 („Identification and characterization of molecular targets in sarcoma cell lines as a basis of innovative therapeutic strategies") zu vergleichen.

Aus durchgeführten Analysen in Teilprojekt 4 („Molecular and translational pathobiology of radiation-induced angiosarcomas") konnte eine neue Klassifikation der Angiosarkome ableitet werden. So zeigen sekundäre Angiosarkome, induziert durch Bestrahlung oder chronische Lymphödeme, c-Myc-Amplikons, während sich diese in primären Angiosarkomen nicht nachweisen lassen. Da Bestrahlungen und Lymphonodektomien derzeit häufig im Rahmen vieler Krebstherapien angewendet werden, erwarten wir einen signifikanten Anstieg der Inzidenz von sekundären Angiosarkomen. Zudem soll in weiteren Studien geprüft werden, ob strahleninduzierte c-myc-Amplifikationen bereits in frühen Läsionen und in strahlengeschädigten Haut- oder Schleimhautbiopsien nachweisbar sind.

Daran angelehnte Studien definierten die Pathogenese von inflammatorischen fibroiden Polypen (IFP) durch die Beschreibung spezifischer aktivierender PDGFRA-Mutationen. Außerdem wurde zum ersten Mal eine neue spezifische Untergruppe von GIST mit $c$-KIT- und

Pathologe 2010 · [Suppl 2] 31:211-214 DOI 10.1007/s00292-010-1340-z

C) Springer-Verlag 2010

A.V. Rüsseler - B. Brors - T. Fischer - J.T. Hartmann - W. Hartmann · P. Hohenberger · P. Lichter · A. Marx · G. Mechtersheimer $\cdot$ R. Penzel · M. Renner $\cdot$ H.-U. Schildhaus $\cdot$ P. Schirmacher · E. Sievers · P. Ströbel · E. Wardelmann · E. Ziesché $\cdot$ R. Büttner

Molekularpathologie von Sarkomen. Erste Ergebnisse des Sarkomforschungsverbundes KoSar

\section{Zusammenfassung}

Zur präzisen Diagnostik von Sarkomen und Behandlung in spezialisierten Zentren hat sich mit Förderung durch die Deutsche Krebshilfe (DKH) der Sarkomforschungsverbund KoSar (Kompetenznetz Sarkome) gebildet. Dieser hat sowohl ein Sarkomgewebearchiv als auch ein Referenzzentrum gegründet, welches mittlerweile etwa 1000 präzise diagnostizierte Sarkome verschiedener Entitäten enthält. Mithilfe dieser Proben konnten u. a. signifikante Genexpressionsprofile für synoviale Sarkome, Leiomyosarkome, myxoide Liposarkome und ein kleines Expressionsprofil für Myxofibrosarkome definiert sowie eine neue Klassifikation der Angiosarkome ableitet werden. Parallel wurden aktivierte Signaltransduktionswege und molekulare Targets für selektive Therapien in Sarkomzelllinien und Xenograft-Transplantations-Modellen identifiziert. Hierauf basierend konnten erste klinische Studien mithilfe der Deutschen Interdisziplinären Sarkom-Studiengruppe (GISG) initiiert werden.

Schlüsselwörter

KoSar - Sarkom - Molekulare Diagnostik ·

Genexpression · Deutsche Interdisziplinäre Sarkom-Studiengruppe

\section{Molecular pathology of sarcomas. Update on the research group "Molecular Diagnosis of Sarcomas"}

\section{Abstract}

To establish precise diagnostic algorithms and standardised treatment of sarcomas in specialized centers, the interdisciplinary research group KoSar (sarcoma competence network) has been funded by German Cancer Aid. A sarcoma tissue repository and a diagnostic reference center have been set up, presently containing about 1000 accurately diagnosed sarcomas of different entities. Significant gene expression profiles for synovial sarcomas, leiomyosarcomas, myxoid liposarcomas and a small profile for myxofibrosarcomas as well as a new classification of an- giosarcomas were defined. We systematically searched for activated signal transduction pathways in sarcoma cell lines and xenograft transplant models and candidate targets for molecular therapies were identified. Based on these results first clinical studies have been initiated by the German Interdisciplinary Sarcoma Study Group (GISG).

Keywords

Research - Sarcoma - Molecular diagnostics . Gene expression · Drug evaluation, preclinical 
PDGFRA-Wildtyp identifiziert, die in ihrer Bedeutung noch unklare homogene NF-1-Deletionen aufweisen (unveröffentlichte Daten). So erwarten wir, dass wir durch kontinuierliche Durchforstung unseres Sarkomarchivs weitere molekular definierte Sarkomentitäten identifizieren können.

Parallel zu den genomweiten Analysen der Sarkomproben sammeln wir alle derzeit verfügbaren Sarkomzelllinien mit charakteristischen genetischen Veränderungen für eine bestimmte Sarkomentität. Alle Teilprojekte (TP 1-4) nutzen systematisch diese Zelllinien, um aktivierende Signaltransduktionswege und Kinasen zu identifizieren. Zudem wird untersucht, ob die Hemmung dieser Signalwege mit einer Wachstumshemmung der Zelllinien korreliert, und Xenograft-TransplantationsModelle wurden etabliert, um potenzielle Zielstrukturen für molekulare Therapien unter präklinischen Behandlungsmodalitäten zu testen.

\section{Etablierung eines klinischen Registers und Initiierung neuer Studien}

Die Durchführung neuer klinischer Studien an Sarkomen bleibt weiterhin ein wichtiges Ziel. Wie zuvor bereits beschrieben, werden die meisten Sarkompatienten außerhalb von spezialisierten Zentren behandelt und daher häufig nicht in Studien rekrutiert. Zur Lösung dieses Problems verfolgen wir zwei Strategien.

Alle klinischen Daten der Patienten, die innerhalb unseres Konsortiums diagnostiziert werden, werden in der CONTICABASE-Datenbank dokumentiert. Diese Datenbank dient zur klinischen Dokumentation in den lokalen Zentren und ermöglicht es uns, die Durchführbarkeit von kollaborativen spezifischen Studien zu evaluieren. Ziel ist es, damit alle klinischen Daten, die Behandlungen und die Follow-up-Daten der Patienten, die in den Plattformen 1 und 2 eingeschlossen sind, zu dokumentieren. Dadurch wird gewährleistet, dass Daten aus dem genetischen und immunhistochemischen Profil mit klinischen Daten korreliert werden können.

Darüber hinaus wurde die Deutsche Interdisziplinäre Sarkom-Studiengrup- pe (GISG, „German Interdisciplinary Sarcoma Group“) als Instrument gegründet, um neue Studien zu initiieren und um die Einbeziehung von Patienten in zurzeit offenen Studien zu fördern. Bei den Fällen, die wir innerhalb unserer Registers aufgreifen, wird empfohlen, sie für chirurgische oder adjuvante Therapieschemata von Peter Reichardt (Bad Saarow), Jörg Thomas Hartmann (Kiel) oder Peter Hohenberger (Mannheim) begutachten zu lassen, damit sie in entsprechende Studien aufgenommen werden können. Der Verbund steht weiterhin in engem Kontakt zu etablierten nationalen Studiengruppen in Deutschland (AIO/IAWS-, CWS/COSS-, EWS-Studiengruppe in Kiel, Stuttgart und Münster).

Erste Studien initiiert von der Deutschen Interdisziplinären Sarkom-Studiengruppe sind:

- GISG I: Behandlung von Patienten mit Desmoidtumoren mit Imatinib,

- GISG II: „First-line-Therapie-Studie“ von Sarkompatienten in der Behandlung mit Paliphosphamid mit oder ohne Adriamycin,

- GISG III: Phase-I/II-Studie bei Sarkompatienten zur Kombination der Strahlentherapie mit oder ohne Sunitinib.

Wir schätzen, dass die Rekrutierung der Patienten im Jahr 2011 abgeschlossen werden kann und die Ergebnisse innerhalb der vorgeschlagenen zweiten Förderperiode des Konsortiums veröffentlicht werden können.

\section{Korrespondenzadresse \\ Dr. A.V. Rüsseler \\ Institut für Pathologie, \\ Universitätsklinikum Bonn \\ Sigmund-Freud-Str. 25, 53127 Bonn \\ vanessa.ruesseler@ukb.uni-bonn.de}

Interessenkonflikt. Die korrespondierende Autorin gibt an, dass kein Interessenkonflikt besteht.

\section{Literatur}

1. Friedrichs N, Küchler J, Endl E et al (2008) Insulinlike growth factor-I receptor acts as a growth regulator in synovial sarcoma. J Pathol 216:428-439

2. Mussi C, Schildhaus HU, Gronchi A et al (2008) Therapeutic consequences from molecular biology for GIST patients affected by neurofibromatosis type I. Clin Cancer Res 14(14):4550-4555
3. Schildhaus HU, Cavlar T, Binot E et al (2008) Inflammatory fibroid polyps harbour mutations in the platelet derived growth factor receptor alpha (PDGFRA) gene. J Pathol 216(2):176-182

4. Rieker RJ, Weitz J, Lehner B et al (2010) Genomic profiling reveals subsets of dedifferentiated liposarcomas to follow separate molecular pathways. Virchows Arch 456(3):277-285

5. Manner J, Radlwimmer B, Hohenberger $P$ et al (2010) MYC high level gene amplification is a distinctive feature of angiosarcomas after irradiation or chronic lymphedema. Am J Pathol 176(1):34-39 